\title{
Quantitative Proteomics of
} Synaptosomal Fractions in a Rat Overexpressing Human DISC1 Gene Indicates Profound Synaptic Dysregulation in the Dorsal Striatum

\author{
Fernando J. Sialana ${ }^{1}$, An-Li Wang ${ }^{2}$, Benedetta Fazari ${ }^{2}$, Martina Kristofova ${ }^{1}$, \\ Roman Smidak ${ }^{1}$, Svenja V. Trossbach ${ }^{3}$, Carsten Korth ${ }^{3}$, Joseph P. Huston ${ }^{2}$, \\ Maria A. de Souza Silva ${ }^{2 *}$ and Gert Lubec ${ }^{4 *}$
}

\begin{abstract}
${ }^{1}$ Department of Pharmaceutical Chemistry, University of Vienna, Vienna, Austria, ${ }^{2}$ Center for Behavioral Neuroscience, University of Düsseldorf, Düsseldorf, Germany, ${ }^{3}$ Department of Neuropathology, Heinrich-Heine University of Düsseldorf, Düsseldorf, Germany, ${ }^{4}$ Department of Neuroproteomics, Paracelsus Private Medical University, Salzburg, Austria
\end{abstract}

OPEN ACCESS

Edited by:

Edna Grünblatt,

Department of Child and Adolescent Psychiatry and Psychotherapy, University of Zurich, Switzerland

Reviewed by: Firas H. Kobeissy, University of Florida, United States Ka Wan Li, VU University Amsterdam, Netherlands

${ }^{*}$ Correspondence: Maria A. de Souza Silva desouza@hhu.de Gert Lubec gert.lubec@/ubeclab.com

Received: 03 November 2017 Accepted: 18 January 2018 Published: 06 February 2018

Citation: Sialana FJ, Wang A-L, Fazari B, Kristofova M, Smidak $R$,

Trossbach SV, Korth C, Huston JP, de Souza Silva MA and Lubec G (2018) Quantitative Proteomics of Synaptosomal Fractions in a Rat Overexpressing Human DISC1 Gene Indicates Profound Synaptic Dysregulation in the Dorsal Striatum.

Front. Mol. Neurosci. 11:26. doi: 10.3389/fnmol.2018.00026
Disrupted-in-schizophrenia 1 (DISC1) is a key protein involved in behavioral processes and various mental disorders, including schizophrenia and major depression. A transgenic rat overexpressing non-mutant human DISC1, modeling aberrant proteostasis of the DISC1 protein, displays behavioral, biochemical and anatomical deficits consistent with aspects of mental disorders, including changes in the dorsal striatum, an anatomical region critical in the development of behavioral disorders. Herein, dorsal striatum of 10 transgenic DISC1 (tgDISC1) and 10 wild type (WT) littermate control rats was used for synaptosomal preparations and for performing liquid chromatography-tandem mass spectrometry (LC-MS)-based quantitative proteomics, using isobaric labeling (TMT10plex). Functional enrichment analysis was generated from proteins with level changes. The increase in DISC1 expression leads to changes in proteins and synaptic-associated processes including membrane trafficking, ion transport, synaptic organization and neurodevelopment. Canonical pathway analysis assigned proteins with level changes to actin cytoskeleton, Gaq, Rho family GTPase and Rho GDI, axonal guidance, ephrin receptor and dopamine-DARPP32 feedback in CAMP signaling. DISC1-regulated proteins proposed in the current study are also highly associated with neurodevelopmental and mental disorders. Bioinformatics analyses from the current study predicted that the following biological processes may be activated by overexpression of DISC1, i.e., regulation of cell quantities, neuronal and axonal extension and long term potentiation. Our findings demonstrate that the effects of overexpression of non-mutant DISC1 or its misassembly has profound consequences on protein networks essential for behavioral control. These results are also relevant for the interpretation of previous as well as for the design of future studies on DISC1.

Keywords: DISC1, proteomics, synapses, animal model, dopaminergic system, axon guidance, striatum 


\section{INTRODUCTION}

Disrupted-in-schizophrenia 1 (DISC1) is a gene originally identified as a translocation mutation in an extended Scottish pedigree where carriers suffered from diverse mental disorders comprising schizophrenia and affective disorders (Millar et al., 2000). Similarly, the DISC1 haplotype was associated with schizophrenia in a Finnish cohort (Hennah et al., 2003). A second family was later identified with a missense mutation and associated diverse clinical phenotypes (Sachs et al., 2005), and genetic association studies have supported association of DISC1 with mental disorders (Chubb et al., 2008). A role of the DISC1 gene for adaptive behavior was also suggested by various animal studies (Brandon and Sawa, 2011; Dahoun et al., 2017).

The DISC1 protein has features of a scaffold protein (Yerabham et al., 2013) and several subdomains have an intrinsic tendency to form high molecular multimers (Yerabham et al., 2017). Insoluble DISC1 protein has been identified in human post mortem brains with mental disorders (Leliveld et al., 2008), indicating that the DISC1 protein can be subject to aberrant proteostasis in vivo. For modeling the effects of aberrant proteostasis in vivo, a transgenic rat model overexpressing (approximately 11-fold) the full length, non-mutant human DISC1 gene (transgenic DISC1, tgDISC1 rat) was generated that exhibited perinuclear aggregates throughout the brain, accentuated in dopamine-rich regions such as in the striatum (Trossbach et al., 2016). The tgDISC1 rat exhibited phenotypes such as amphetamine supersensitivity, an increase in D2Rhigh receptors, and dopamine transporter mislocalization and dysfunction consistent with phenotypes observed in schizophrenia (Trossbach et al., 2016). Also, at the neuroanatomical level fewer dopaminergic neurons and projections into the dorsal striatum, as well as aberrant interneuron positioning was observed indicating subtle neurodevelopmental disturbance (Hamburg et al., 2016).

These findings, induced by aberrant proteostasis of the DISC1 protein, leading to its misassembly and perinuclear deposition, suggest an important role of the DISC1 protein and its correct assembly for protein networks involved in adaptive behavior. Such protein networks have been described both, at the protein and the genetic level. At the genetic level, Teng et al. (2017) carried out targeted sequencing of 59 DISC1 interactome genes and 154 regulome genes in psychiatric patients, identifying altered regulation of schizophrenia candidate genes by DISC1. In an attempt to dissect DISC1 function through proteinprotein interactions based upon a yeast two-hybrid system along with bioinformatic methods, a comprehensive network around DISC1 was generated (Camargo et al., 2007). Using this iterative yeast two-hybrid system, a framework was provided to explore the function of DISC1, and interrogation of the proposed interactome has shown DISC1 to have proteinprotein interactions consistent with that of an essential synaptic protein (Camargo et al., 2007). Current evidence suggests that DISC1 functions as a neuronal intracellular trafficking regulator that includes transport of neurotransmitter receptors, vesicles, mitochondria and mRNA, rendering synaptic regulation vulnerable to DISC1 dysfunction (Devine et al., 2016).
The objective of this study was to identify the proteomic signatures of the tgDISC1 rat model vs. its littermate wild type (WT) control to gain insights onto the DISC1-regulated proteins and downstream synaptic processes and to identify molecular circuitry regulated by relatively modest changes in expression level leading to DISC1 misassembly. Identification of changes in protein networks relevant for behavioral processes would raise the possibility for the DISC1 protein to represent a non-genetic interface with exogenous influences for mental disorders.

There is mounting evidence for a focal role of the DISC1 protein in striatal functions, and particularly on dopamine homeostasis in relation to behavioral changes (Trossbach et al., 2016; Wang et al., 2017). Therefore we chose to select proteins from the synapse-enriched membrane fractions (synaptosomes) from the dorsal striatum for this study. Differential proteomics by isobaric labeling (TMT10plex) enable multiplexed protein identification and quantitative analysis by liquid chromatography-tandem mass spectrometry (LC-MS/MS). This allows the unbiased analyses of approximately 6000 proteins and targets synaptic proteins including receptors, transporters and channels that have been implicated in psychiatric disorders. Combining proteomics and bioinformatics approaches enabled a comprehensive view on the in vivo protein changes and the biological functions of DISC1.

\section{MATERIALS AND METHODS}

\section{Animals}

Previously described tgDISC1 Sprague-Dawley rats and WTs were used in this study (Trossbach et al., 2016). Briefly, full-length, non-mutant human DISC1 as transgene with the polymorphisms $\mathrm{F} 607$ and C704 were integrated into the pronuclei of Sprague Dawley rats. Ten male tgDISC1 rats and 10 male WT littermate control rats, aged 14-15 months (ZETT, Heinrich Heine University, Düsseldorf, Germany) were used. One WT rat and one tgDISC1 rat were derived from each pair of parents. The study was carried out in accordance with the "Principles of laboratory animal care" (NIH publication No. 86-23, revised 1985), and the German Law on the Protection of Animals. It was approved by the Landesamt für Natur, Umwelt und Verbraucherschutz (LANUV) NRW.

\section{Preparation of Synaptosomal Fractions}

Dorsal striata from fresh brains were dissected and stored at $-80^{\circ} \mathrm{C}$. Synaptosomal fractions from bilateral regions were prepared for individual animals (for tgDISC1 and WT; $n=10$ each), using a microscale discontinuous sucrose gradient modified from previous protocols (Hahn et al., 2009; Sialana et al., 2016). Collected synaptosomes from 1.25/1.0 M sucrose interface were diluted with $10 \mathrm{mM}$ HEPES, divided into two and pelleted at $15,000 \times g$ for $30 \mathrm{~min}$. Pelleted synaptosomal samples were reconstituted in urea buffer $(7 \mathrm{M}$ urea, $2 \mathrm{M}$ thiourea, 4\% CHAPS, $100 \mathrm{mM}$ DTT, $50 \mathrm{mM}$ TEAB supplemented with protease inhibitors) for LCMS analyses and SDS buffer 
(1.5\% SDS, $100 \mathrm{mM} \mathrm{NaCl}, 20 \mathrm{mM}$ Tris supplemented with protease inhibitors) for WB analyses and were sonicated for $1 \mathrm{~h}$. Protein amounts were estimated using the Pierce 660 protein assay or BCA protein assay (ThermoFisher Scientific).

\section{Proteolytic Digestion and Isobaric Labeling}

Fifty micrograms of samples were digested with a Trypsin-LysC enzyme mixture $(1: 100 \mathrm{w} / \mathrm{w}$, Promega) using the filter-aided sample preparation (FASP), as previously described, with minor modifications (Wisniewski et al., 2009). The resulting peptide samples were purified with reversed-phase C18 and labeled with TMT 10-plex according to the instructions supplied by the manufacturer. Two TMT-10plex experiments were performed, with each experiment consisting of five tgDISC1 and five WT animals $(n=10$ biological replicates per group). For each TMT experiment, ten isobarically labeled peptide samples were pooled, the peptides separated by high $\mathrm{pH}$ reversed-phase LC into 100 time-based fractions and pooled into 25 samples (Gilar et al., 2005). The peptides were vacuum concentrated and reconstituted in 5\% formic acid. Details of the procedure are essentially as described previously (Sialana et al., 2016) and in the Supplementary Figure S1.

\section{Liquid Chromatography and Tandem Mass Spectrometry}

Samples were injected onto a Dionex Ultimate 3000 system (ThermoFisher Scientific) coupled to a Q-Exactive Plus mass spectrometer (ThermoFisher Scientific, Schwerte, Germany). Software versions used for the data acquisition and operation of the Q-Exactive were Tune 2.8.1.2806 and Xcalibur 4. HPLC solvents were as follows: solvent A consisted of $0.1 \%$ formic acid in water and solvent B consisted of $0.1 \%$ formic acid in $80 \%$ acetonitrile. From a thermostated autosampler, $10 \mu \mathrm{L}$ that correspond to $1 \mu \mathrm{g}$ of the peptide mixture were automatically loaded onto a trap column (PM100-C18 $3 \mu \mathrm{m}$, $75 \mu \mathrm{m} \times 20 \mathrm{~mm}$, ThermoFisher Scientific, Austria) with a binary pump at a flow rate of $5 \mu \mathrm{L} / \mathrm{min}$ using $2 \%$ acetonitrile in $0.1 \%$ TFA for loading and washing the pre-column. After washing, the peptides were eluted by forward-flushing onto a $50 \mathrm{~cm}$ analytical column with an inner diameter of $75 \mu \mathrm{m}$ packed with $2 \mu \mathrm{m}-\mathrm{C} 18$ reversed phase material (PepMap-C18 $2 \mu \mathrm{m}, 75 \mu \mathrm{m} \times 500 \mathrm{~mm}$, ThermoFisher Scientific, Austria). For label free quantification (LFQ), the LCMS analyses was performed using a single-shot LCMS approach with 4-h gradient with LCMS parameters as described previously (Stojanovic et al., 2017).

The fractionated TMT10plex labeled peptides were eluted from the analytical column with a 120 min gradient ranging from $5 \%$ to $37.5 \%$ solvent $\mathrm{B}$, followed by a 10 min gradient from $37.5 \%$ to $50 \%$ solvent B and finally, to $90 \%$ solvent B for $5 \mathrm{~min}$ before re-equilibration to $5 \%$ solvent $\mathrm{B}$ at a constant flow rate of $300 \mathrm{~nL} / \mathrm{min}$. The LTQ Velos ESI positive ion calibration solution (Pierce, IL, USA) was used to externally calibrate the instrument prior to sample analysis and an internal calibration was performed on the polysiloxane ion signal at $\mathrm{m} / \mathrm{z}$ 445.120024 from ambient air. $\mathrm{MS}^{1}$ scans were performed from $\mathrm{m} / \mathrm{z} 375-1400$ at a resolution of 70,000. Using a data-dependent acquisition mode, the 15 most intense precursor ions of all precursor ions with +2 to +7 charge were isolated within a $1.2 \mathrm{~m} / \mathrm{z}$ window and fragmented to obtain the corresponding MS/MS spectra. The fragment ions were generated in a higherenergy collisional dissociation (HCD) cell at 32\% normalized collision energy with a fixed first mass at $100 \mathrm{~m} / \mathrm{z}$ and detected in an Orbitrap mass analyzer at a resolution of 35,000 . The dynamic exclusion for the selected ions was $30 \mathrm{~s}$. Maximal ion accumulation time allowed in MS and $\mathrm{MS}^{2}$ mode was 50 and $100 \mathrm{~ms}$, respectively. Automatic gain control was used to prevent overfilling of the ion trap and was set to $3 \times 10^{6}$ ions and $1 \times 10^{5}$ ions for a full Fourier transform $\mathrm{MS}$ and $\mathrm{MS}^{2}$ scan, respectively.

\section{Protein Identification and Quantification}

All MS-MS ${ }^{2}$ spectra were searched against UniProtKB/SwissProt rat protein database version v 2016.04.14 (27,815 sequences, including isoforms). In addition, sequences of the human DISC1 protein and 11 isoforms produced by alternative splicing with the polymorphisms F607 and C704 were appended to the rat database. All spectra files were processed in Proteome Discoverer 2.1 (Thermo Scientific, Germany) platform with Mascot using mass tolerances of $\pm 10 \mathrm{ppm}$ and $\pm 0.02 \mathrm{Da}$ for precursor and fragment ions. One missed tryptic cleavage site was allowed. Oxidation of methionine was set as variable modification, whilst carbamidomethylation of cysteine residues, TMT 10-plex labeling of peptide N-termini and lysine residues were set as fixed modification. Thresholds were determined via the target-decoy approach using a reversed protein database as the decoy by imposing $1 \%$ false discovery rate (FDR). Label-free quantitation was implemented using the Minora feature of Proteome Discoverer 2.2. The following parameters are used: maximum retention time alignment of $10 \mathrm{~min}$ with minimum of $\mathrm{S} / \mathrm{N}$ of 5 for feature linking mapping. Abundance were based precursor/peptide area intensities. Normalization was performed such that the total sum of the abundance is the same for all sample channels. Imputation was performed by replacing the missing values with random values from the lower $5 \%$ of the detected values. For TMT 10-plex labeled samples, relative abundances of proteins were determined from the TMT reporter ions without imputation. Protein abundance ratios were calculated based on unique and razor peptides. Relative protein levels were determined from the sum of the reporter ion intensities per quantitative channel that correspond to each biological animal replicate.

The MS proteomics data have been deposited to the ProteomeXchange Consortium via the PRIDE (Vizcaíno et al., 2014) partner repository with the dataset identifier PXD008123.

\section{Bioinformatics}

Quantitative data were analyzed using Perseus statistical package (version 1.5.1.6; Tyanova et al., 2016). Statistical significance of differences in protein levels between the groups were evaluated using a two-sided $T$-test with $P<0.05$ (either Student's or Welch's as required). Enrichment of GO annotations were 

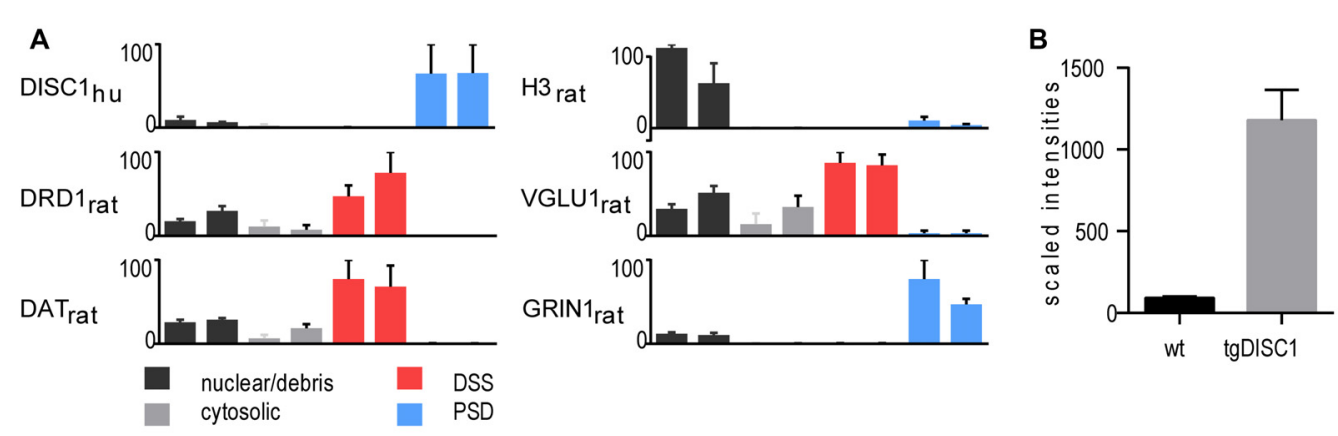

FIGURE 1 | Proteomic profile of the transgenic DISC1 (tgDISC1) fractions. (A) Protein levels of representative proteins for the following biochemical fractions of the dorsal striatum: nuclear/debris, cytosolic, detergent-soluble synaptosomal preparation (DSS), postsynaptic density (PSD) preparation. Protein levels of representative synaptic markers were estimated from label-free LCMS analyses. Protein levels of the nuclear (H3), presynaptic (VGLU1) and postsynaptic (GRIN1) protein markers are enriched in nuclear/debris, DSS and PSD preparations, respectively. The majority of the human Disrupted-in-schizophrenia 1 (DISC1) protein was enriched in the PSD preparations. The level of overexpression is approximately 10-fold higher than the endogenous DISC1 protein in the whole synaptosomes (B).

performed on the significant proteins using GOA database (v30.08.2017) using the ClueGO via the Cytoscape platform (Bindea et al., 2009; Huntley et al., 2015). To reduce redundancy of GO terms the fusion option was selected. Enriched GO terms (Benjamini-Hochberg $P$-value $<0.05$ ) are functionally grouped into networks linked by their kappa score level $(\geq 0.40)$. Functionally related groups partially overlap and only the most significant terms per group are labeled. Pathway analyses on the significant proteins were performed through the use of IPA (Ingenuity ${ }^{\circledR}$ Systems ${ }^{1}$ ). The differentially expressed genes were categorized to related canonical pathways. Only those experimentally observed or highly predicted molecules and/or relationships from tissues and cells from the nervous system were considered. The top enriched categories of canonical pathways with a $P$-value $<10^{-3}$ as well as representative differentially expressed proteins in each canonical pathway is reported. Curated gene-disease annotations were obtained from Comparative Toxigenomics database (Davis et al., 2015). The IPA regulation $z$-score algorithm was used to predict biological functions that are expected to be activated ( $z$-score $\geq 2 ; P \leq 0.05$ ). The $z$-scores take into account the directional effect of one protein on a process and the direction of change of molecules in the dataset.

\section{Immunoblotting}

The following antibodies were used according to the instructions supplied by the manufacturer: mouse anti-PSD95 (124011, Synaptic Systems), mouse anti-SYP (sc-55507, Santa Cruz Biotechnology), rabbit anti-NMDAR1 (ab32915, Abcam), mouse anti-VGLUT1 (135311, Synaptic Systems), rabbit anti-GAPDH (ab9485, Abcam), rabbit anti-DAT1 (ab111468, Abcam) and mouse anti-huDISC1 (3D4, Korth lab; Ottis et al., 2011). Immunoblot data were normalized to corresponding whole-lane densitometric volumes of protein-stained membranes (Welinder and Ekblad, 2011). Immunoblotting conditions were as

\footnotetext{
${ }^{1}$ www.ingenuity.com
}

previously described (Sialana et al., 2016) and antibody dilutions are provided in the Supplementary Table S1.

\section{RESULTS}

In the current study, a high-throughput proteomic approach was employed to generate a comprehensive view of the in vivo protein changes in striatal synaptosomes of the tgDISC1 rat model (experimental workflow, Supplementary Figure S1). Methodologically, tissue fractionation was initially performed on the dorsal striatum of tgDISC1 rats to determine the subcellular expression of tgDISC1 and which enrichment steps would be employed in this study (Phillips et al., 2001; Sialana et al., 2016). Dorsal striata of tgDISC1 rats were fractionated into nuclear/debris, cytosolic, detergent soluble synaptosome (DSS) and postsynaptic density (PSD) preparations. LCMS-based proteomic analyses of the biochemical fractions resulted in the identification and LFQ of 5002 protein groups (Supplementary Data 1). Distribution of the nuclear (H3), presynaptic (VGLU1) and postsynaptic (GRIN1) protein markers enriched in nuclear/debris, DSS and PSD preparations is given in Figure 1A. Although DISC1 was observed in all preparations, the majority of the human DISC1 protein was enriched in the TritonX100-resistant PSD fractions. This is in agreement with previous immunoblotting studies of DISC1 in adult rats (Hayashi-Takagi et al., 2010). We have previously shown that dopaminergic pathways are modulated in the striatum of the tgDISC1 rat (Trossbach et al., 2016). Taking into account that dopamine receptor 1 and the dopamine transporter were highly enriched in the DSS preparations (Figure 1A), it was decided to study the whole synaptosome for quantitative proteomics experiments. Immunoblots of postsynaptic (GRIN1 and PSD95) and presynaptic (VGLU1 and SYP) proteins show enrichment of synaptosomal proteins on the biochemical fraction (Supplementary Figure S2). The level of overexpression is approximately 10-fold higher than 


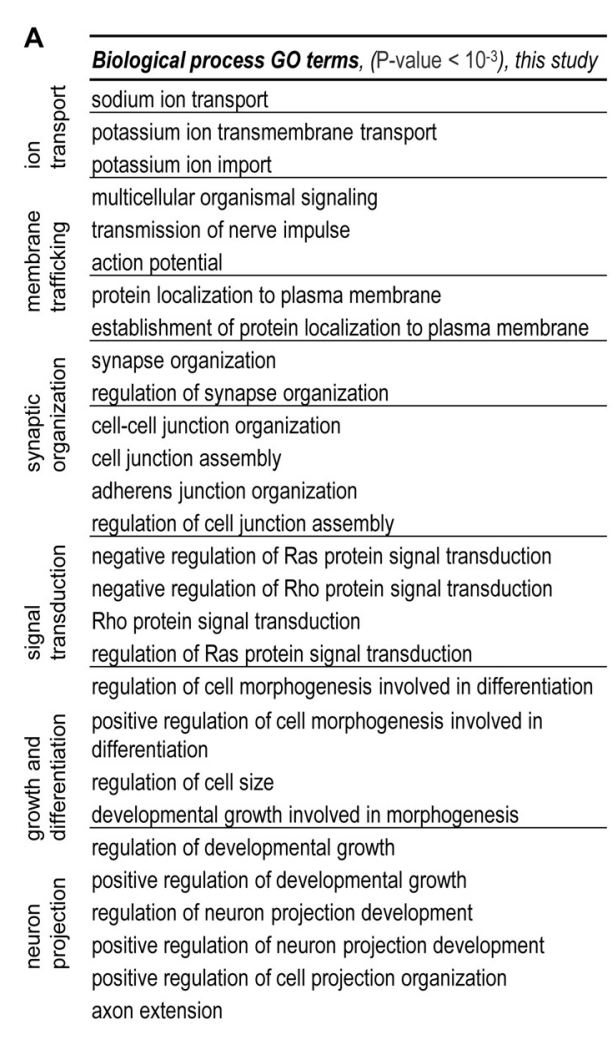

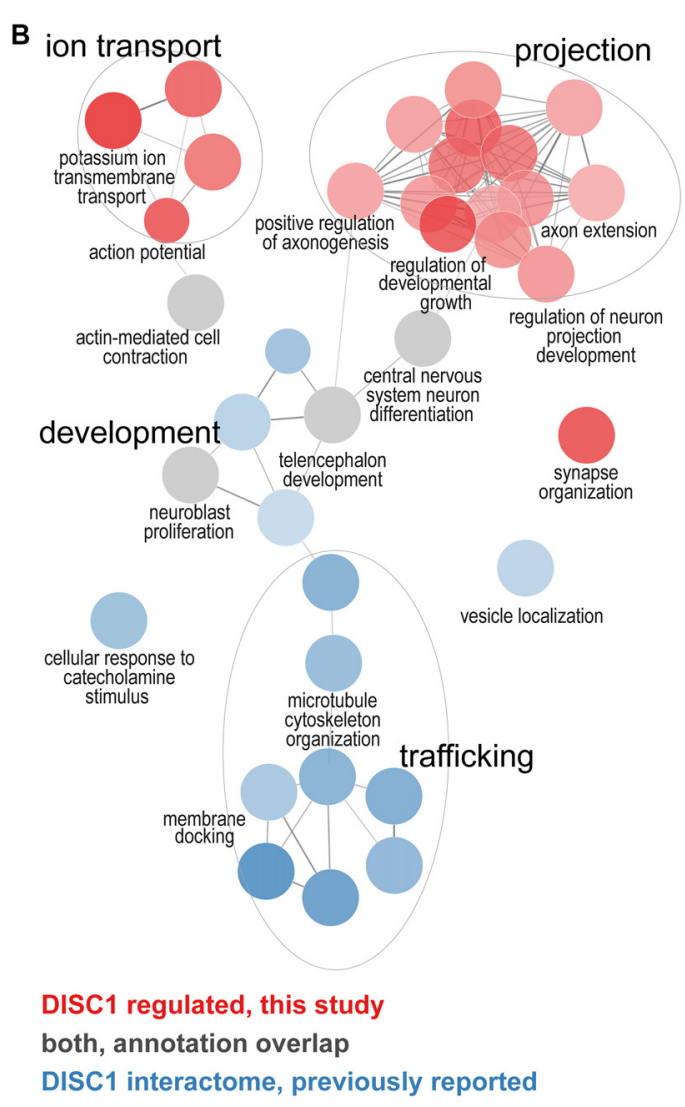

FIGURE 2 | (A) Biological processes regulated by DISC1. Enriched GO terms $\left(P\right.$-value $\left.<10^{-3}\right)$ are functionally grouped. The synaptic components terms as well as membrane trafficking, ion transport, synaptic organization and neurodevelopment processes are well represented. (B) Comparison of the DISC1-regulated proteins and the previously reported interacting proteins. In the current study ion transport, projections and synaptic organization were novel findings. Developmental processes from previous studies were confirmed (gray GO enrichment analyses was performed using ClueGO. Enriched GO terms (Benjamini-Hochberg $P$-value $<10^{-3}$ ) are functionally grouped into networks linked by their kappa score level $(\geq 0.40)$. Functionally related groups partially overlap and only the most significant terms per group are labeled.

the endogenous DISC1 protein in the whole synaptosomes (Figure 1B).

\section{DISC1 Regulated Proteins-Proteomic Profiling of Striatal Synaptosomes}

An expression proteomics experiment was performed to identify the proteins potentially regulated by DISC1. Synaptosomal fractions of bilateral dorsal striata of $10 \mathrm{wt}$ and $10 \mathrm{tgDISC} 1$ rats using TMT10plex were analyzed in two separate 10-plex experiments (5 tgDISC1 and 5 WT). In total, 7227 protein groups were identified (Supplementary Data 2) including 252 receptors and 672 transporters/channels. Out of the 6153 quantifiable protein groups, 213 proteins were statistically different between the tgDISC1 and WT rats (Supplementary Table S2, Supplementary Data 3). Protein levels were considered statistically different between groups when $P \leq 0.05$ using a two-sided T-test (either Student's or Welch's as required). Given the large number of comparisons made and the possibility of Type 1 error, the $p$ values given cannot be interpreted in terms of "significance", but rather as "measures of effect".
As we used a good number of biological replicates for TMT-based proteomics (10 animals per group), we opted to use $T$-test that performs "individual proteinsbased" hypotheses test (T-test) rather than a background "all-proteins-based" hypothesis test (FDR). TMT-based proteomics experiments are sensitive and precise but quantification is known to undergo ratio compression (Ow et al., 2011). The values from FDR corrections depend on effect size; smaller differences yield higher P-corrected (q-values); thus only two proteins passed the corrected thresholds. An additional filter is applied when enrichment analyses (GO annotation, IPA) is employed. Slight differences in the levels of multiple proteins should cluster relevant processes and the proteins from the top enriched processes/pathways are of higher emphasis (Pascovici et al., 2016).

Immunoblotting analyses of DAT1, GRIN1 and DISC1 of WT and tgDISC1 indicated that the direction of fold differences measured by TMT-proteomics and western blotting (Supplementary Figure S3) was consistent. 
A

\begin{tabular}{|c|c|c|}
\hline $\begin{array}{ll}\text { Canonical Pathways } \\
\end{array}$ & $P$-value & Proteins \\
\hline Signaling by Rho Family GTPases & $4.57 \mathrm{E}-06$ & $\begin{array}{l}\text { MYL6, ARHGEF4, RAF1, ROCK1, GNAZ, FGFR3, DES, PIK3CA, } \\
\text { ARPC1B, CDH20, GNAS, ITGA3 }\end{array}$ \\
\hline Axonal Guidance Signaling & $1.07 \mathrm{E}-04$ & $\begin{array}{l}\text { MYL6, LINGO1, RAF1, ABLIM2, EPHA6, ROBO2, ROCK1, GNAZ, } \\
\text { FGFR3, SEMA7A, PIK3CA, ARPC1B, GNAS, ITGA3 }\end{array}$ \\
\hline RhoGDI Signaling & 2.57E-04 & MYL6, ARHGEF4, ROCK1, GNAZ, ARPC1B, CDH20, GNAS, ITGA3 \\
\hline Ephrin Receptor Signaling & 2.69E-04 & RAF1, EPHA6, ROCK1, GNAZ, ARPC1B, GNAS, ITGA3, GRIN1 \\
\hline Actin Cytoskeleton Signaling & 3.47E-04 & $\begin{array}{l}\text { MYL6, ARHGEF4, RAF1, ROCK1, CSK, FGFR3, PIK3CA, ARPC1B, } \\
\text { ITGA3 }\end{array}$ \\
\hline Gaq Signaling & $8.71 \mathrm{E}-04$ & RAF1, ADRA1A, ROCK1, CSK, FGFR3, PIK3CA, GNAS \\
\hline $\begin{array}{l}\text { Dopamine-DARPP32 Feedback in cAMP } \\
\text { Signaling }\end{array}$ & $9.33 \mathrm{E}-04$ & CACNA1A, ADCY3, KCNJ4, KCNJ11, GNAS, KCNJ12, GRIN1 \\
\hline
\end{tabular}

B DISC1 regulates axonal guidance signaling receptors
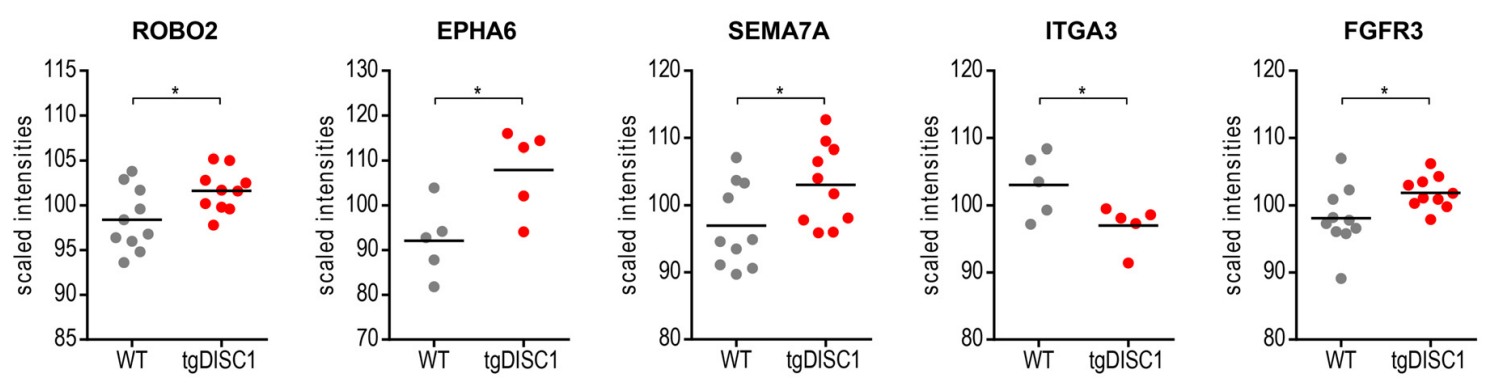

C Regulation of the dopaminergic sytem in DISC1 overexpressed rats
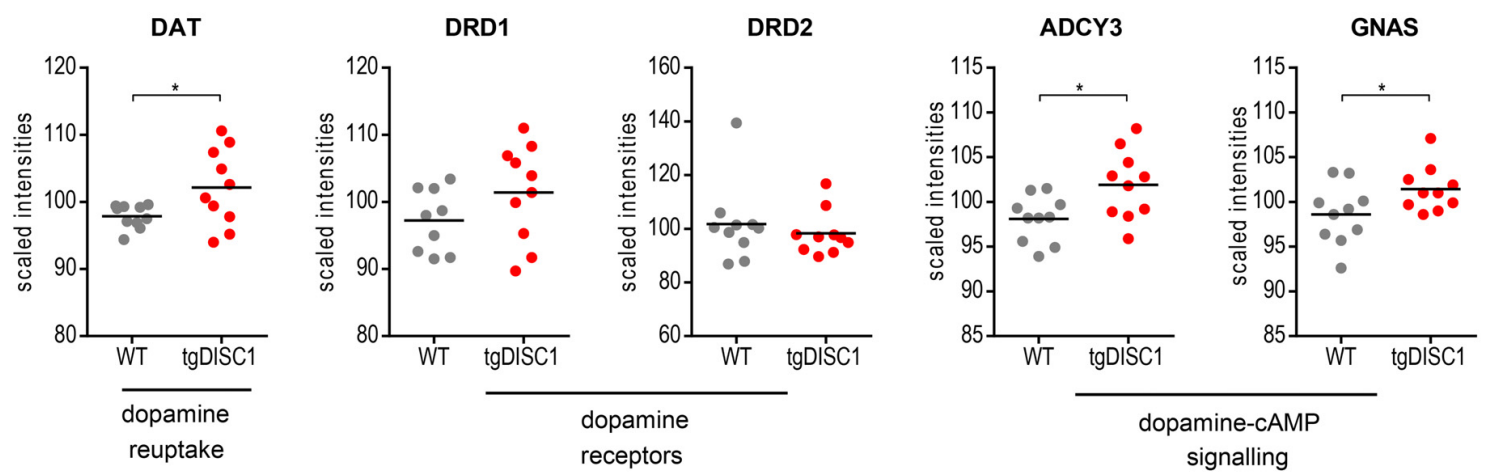

FIGURE 3 | Pathways regulated by DISC1. Significantly enriched canonical pathways (Fishers' exact test, $P<10^{-3}$, IPA) of the proteins altered in by tgDISC1 rats in the dorsal striatum (A). Representative proteins from the dopaminergic (B) and axonal guidance signaling pathway (C) are shown. Values represent ${ }^{*} p<0.05$,

${ }^{* *} p<0.01,{ }^{* * *} p<0.001$ compared using two-sided $T$-tests.

\section{Functional Classification of Proteins Modulated in tgDISC Rats}

The biological functions of the 213 proteins with highly different protein level changes between wildtype and tgDISC1 rats were explored using GO enrichment analyses. Enrichment of synaptic components such as axons, dendritic spines, membrane rafts, neuron projection membrane, and the ion channel complex were revealed (Supplementary Table S3, Supplementary Figure S4). The voltage gated ion channels were the major protein classes represented (Supplementary Table S4). The results suggest that the modest overexpression of the full-length human DISC1 alters proteins linked to synaptic processes including membrane trafficking, ion transport, synaptic organization and neurodevelopment (Figure 2A).

\section{Functional Comparison of the DISC1 Regulated Proteins to Known Interacting Proteins}

To determine the biological functions unique to DISC1 regulated proteins, we performed enrichment analyses for the 


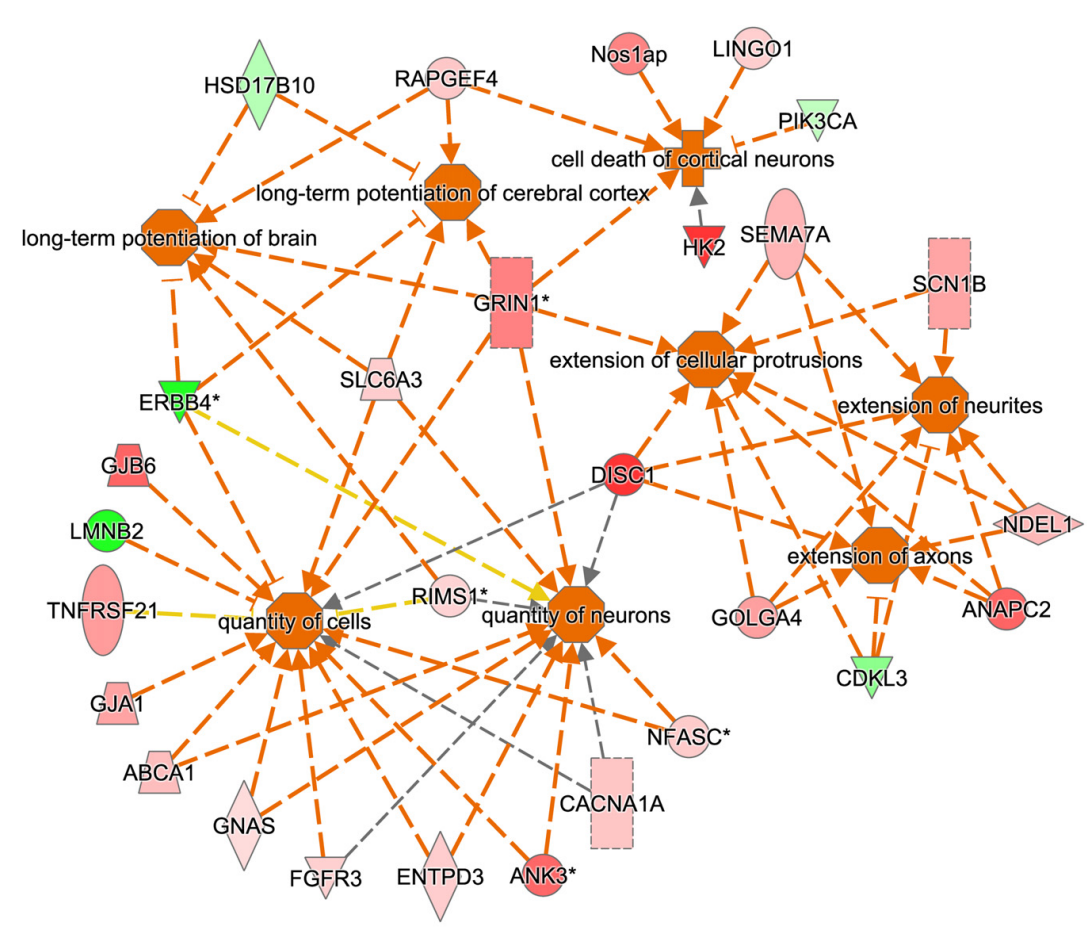

FIGURE 4 | Predicted biological functions of the tgDISC1 regulated proteins as evaluated by IPA. The IPA regulation z-score algorithm was used to predict the activation of biological functions in tgDISC1 rats relative to wild type (WT) according to our proteomics data. The network displays functional interactions between proteins $(z$-score $\geq 2$ and $p$-value $\leq 0.05)$. Dashed lines indicate direct or indirect interactions. Proteins up-regulated in tgDISC1 rats are colored in shades of red; proteins down/regulated are colored in green.

DISC1 regulated proteins in comparison to previously reported interacting proteins (Camargo et al., 2007; Boxall et al., 2011; Bradshaw and Porteous, 2012; Thomson et al., 2013) as compiled by a recent study (Teng et al., 2017). Using ClueGO, 36 biological processes with strong enrichment $\left(P<10^{-6}\right)$ were revealed (Figure 2B; Supplementary Figure S5). The clusters of biological processes exclusive to the proteins regulated by DISC1 include: "regulation of neuron projection development", "positive regulation of axonogenesis", "action potential/potassium ion transport and synapse organization". Terms associated with microtubule development and neuronal transport were highly represented in the DISC1-interacting proteins. Biological processes such as "CNS differentiation" and "telencephalon development" were enriched in both, DISC1 regulated and interacting protein data sets.

\section{Prediction of Canonical Pathways and Biological Function}

To investigate the molecular mechanisms modulated by DISC1, data were analyzed through the use of Ingenuity Pathway analysis (IPA; Ingenuity ${ }^{\circledR}$ Systems ${ }^{2}$ ). The differentially expressed proteins were categorized to related canonical pathways. Canonical pathway analysis assigned proteins with

${ }^{2}$ www.ingenuity.com level changes to actin cytoskeleton, Gaq, Rho family GTPase and Rho GDI-, axonal guidance, ephrin receptor and dopamineDARPP32 feedback in cAMP signaling (Fisher's exact test, $P<10^{-3}$, Figure 3A, Supplementary Figure S6). Only robustly predicted or experimentally observed molecules and/or relationships from tissues and cells from the nervous system were considered. Receptors from the axonal guidance signaling and the dopamine-DARPP32 feedback from the cAMP signaling canonical pathway are illustrated in Figures 3B,C).

The IPA regulation $z$-score algorithm was used to predict biological functions that are expected to be activated in tgDISC1 rats rather than in wildtype (positive $z$-score) according to own proteomics data $(z$-score $\geq 2 ; P \leq 0.05)$. The $z$-scores take into account the directional effect of one protein on a process and the direction of change of molecules in the dataset. From the expression data of the regulated proteins, the following processes are predicted to be activated: "activation regulation of cell quantities", "neuronal and axonal extension", "long term potentiation" and "apoptosis" (Figure 4, Supplementary Table S5).

Annotation of the DISC1 altered protein levels revealed that 54 proteins are associated with mental disorders and/or nervous system diseases as implemented by the Comparative Toxicogenomics Database (CTD; Davis et al., 2015). Disease-gene associations were based on genomic, transcriptomic and proteomic studies on the sequence variation 
and expression changes associated with brain diseases and disorders. Over-represented disease-protein associations (Fishers' exact test, $P<0.05$ ) include: neurodevelopmental disorders, autistic disorders, schizophrenia spectrum, anxiety disorders, substance-related disorders (e.g., cocaine) and intellectual disability (Table 1). In particular, the schizophreniaassociated proteins including dopamine transporter 1 (SLC6A3), receptor tyrosine-protein kinase erbB-4 (ERBB4), glutamate ionotropic receptor NMDA type subunit 1 (GRIN1), membrane associated guanylate kinase $\mathrm{WW}$ and $\mathrm{PDZ}$ domain containing 2 (MAGI2) and regulator of G-protein signaling 12 (RGS12) were also regulated by DISC1 (Mateos et al., 2006; Silberberg et al., 2006; Xu et al., 2011; Koide et al., 2012; Guipponi et al., 2014; Jaros et al., 2015; Zhang et al., 2015; Li et al., 2017).

\section{DISCUSSION}

By the use of quantitative proteomics of synapse-enriched membrane (synaptosome) fractions of the dorsal striatum of the tgDISC1 rat, we have identified novel protein networks and signaling pathways regulated by an increase of non-mutant DISC1 expression or DISC1 misassembly. These results suggest that the DISC1 protein and its disturbed proteostasis can have an effect on mental disorder-relevant protein networks independent of genetic mutations. Likely, multiple exogenous or endogenous factors other than overexpression could lead to a failure of DISC1 proteostasis, such as exposure to high dosages of dopamine or other oxidants, making DISC1 protein an oxidation “sensor" (Atkin et al., 2012; Trossbach et al., 2016).

In the tgDISC1 rat, an about 11-fold overexpression, leading to DISC1 misassembly, changed proteins and synapticassociated processes including membrane trafficking, ion transport, synaptic organization and neurodevelopment is observed. Furthermore, dysregulation of DISC1 potentially modulates pathways including actin cytoskeleton, G $\alpha \mathrm{q}$, Rho family GTPase and Rho GDI-, axonal guidance, ephrin receptor and dopamine-DARPP32 feedback in cAMP signaling associated with the synaptic pathologies. DISC1-regulated proteins are also highly associated with neurodevelopmental disorders, autistic disorder, schizophrenia spectrum, anxiety disorders, substancerelated disorders and intellectual disability (Figure 5).

Previously known DISC1-protein interactors have been reported to modulate synaptic processes. The current study revealed that DISC1 regulates an array of synaptic proteins and processes that complements previous protein interaction results (Supplementary Figure S7). Proteins that were previously reported to interact with DISC1 (Millar et al., 2003; Camargo et al., 2007) were also modified in the current study in the tgDISC1 rat. These include microtubule proteins pericentrin (PCNT), GRIP1 associated protein 1 (GRIPAP1), microtubule associated protein 1A (MAP1A), nudE neurodevelopment protein 1 (NDEL1) and microtubule-actin crosslinking factor 1 (MACF1) that are involved in neuronal cytoskeleton organization and membrane transport processes.

Dysregulation of DISC1 was reported to modulate glutamatergic and dopaminergic systems as previously reviewed (Hayashi-Takagi et al., 2010; Ramsey et al., 2011; Dahoun et al., 2017). Own results herein show that NMDAR1 is increased in the striatum of the tgDISC1 rat. A relationship between NMDAR1 and DISC1 has been shown, as knockdown and antagonists of NMDAR1 reduced numbers of synapses and synaptic DISC1 mainly in the striatum (Ramsey et al., 2011). Further, the DISC1 interactor GRIPAP1 is increased in the tgDISC1 rat. GRIPAP1 controls the AMPA receptors/GRIPcomplex transport to the synapse by NMDA receptor activation (Ye et al., 2000).

As shown by MS, dopamine transporter levels were highly increased in the tgDISC1 rats, consistent with own previous studies by immunoblotting (Trossbach et al., 2016). Whereas levels of dopamine receptors 1 and 2 were not significantly altered, pathway enrichment analyses (Figure 3C) suggest that proteins (e.g., ADCY3, GNAS) from the dopamineDARPP32 feedback of the cAMP signaling canonical pathway, may be involved in modulation of the known dopaminergic deficits in tgDISC1. Adenylate cyclase ADCY3 as a downstream effector of dopaminergic pathways catalyzes the formation of cAMP in response to G-protein signaling.

TABLE 1 | Disease-protein association of the DISC1 regulated proteins.

\begin{tabular}{|c|c|c|}
\hline Disease name & $P$-value & Proteins \\
\hline Neurodevelopmental disorders & $1.02 \mathrm{E}-07$ & $\begin{array}{l}\text { ANK3, ASIC2, CADM1, CTTNBP2, DISC1, GJA1, GNAS, GRIN1, KCNA2, KCNMA1, } \\
\text { RIMS1, ROBO2, SCN2A, SLC4A4, SLC6A3, STAMBP, TCN2 }\end{array}$ \\
\hline Mental disorders & 5.11E-07 & $\begin{array}{l}\text { ANK3, ASIC2, CADM1, CTTNBP2, DISC1, GC, GJA1, GNAS, GRIN1, KCNA2, } \\
\text { KCNMA1, KLHL5, LINGO2, MAGI2, RGS12, RIMS1, ROBO2, SCN2A, SLC4A4, } \\
\text { SLC6A3, STAMBP, TCN2 }\end{array}$ \\
\hline Autistic disorder & 3.40E-05 & ASIC2, CADM1, DISC1, GJA1, KCNMA1, RIMS1, ROBO2, TCN2 \\
\hline Schizophrenia spectrum and other psychotic disorders & 4.10E-04 & DISC1, GC, GRIN1, MAGI2, RGS12, SLC6A3 \\
\hline Anxiety disorders & 2.53E-02 & MAGI2, SLC6A3 \\
\hline Cocaine-related disorders & $1.74 \mathrm{E}-02$ & GRIN1, KLHL5, SLC6A3 \\
\hline Intellectual disability & 2.52E-02 & ANK3, DISC1, GNAS, GRIN1, KCNA2, SLC4A4 \\
\hline Psychotic disorders & 1.13E-02 & GRIN1, SLC6A3 \\
\hline Schizophrenia & $1.62 \mathrm{E}-03$ & DISC1, GC, MAGI2, RGS12, SLC6A3 \\
\hline Substance-related disorders & 3.98E-02 & GNAS, GRIN1, KLHL5, LINGO2, SLC6A3 \\
\hline
\end{tabular}

Gene-disease associations on the DISC1 regulated proteins were implemented in the Comparative Toxicogenomics Database, CTD. Fifty-four DISC1-regulated proteins are associated with mental disorders and/or nervous system disease disorders. Over-represented disease-protein associations (Fishers' exact test, $P$ < 0.05) are illustrated. 


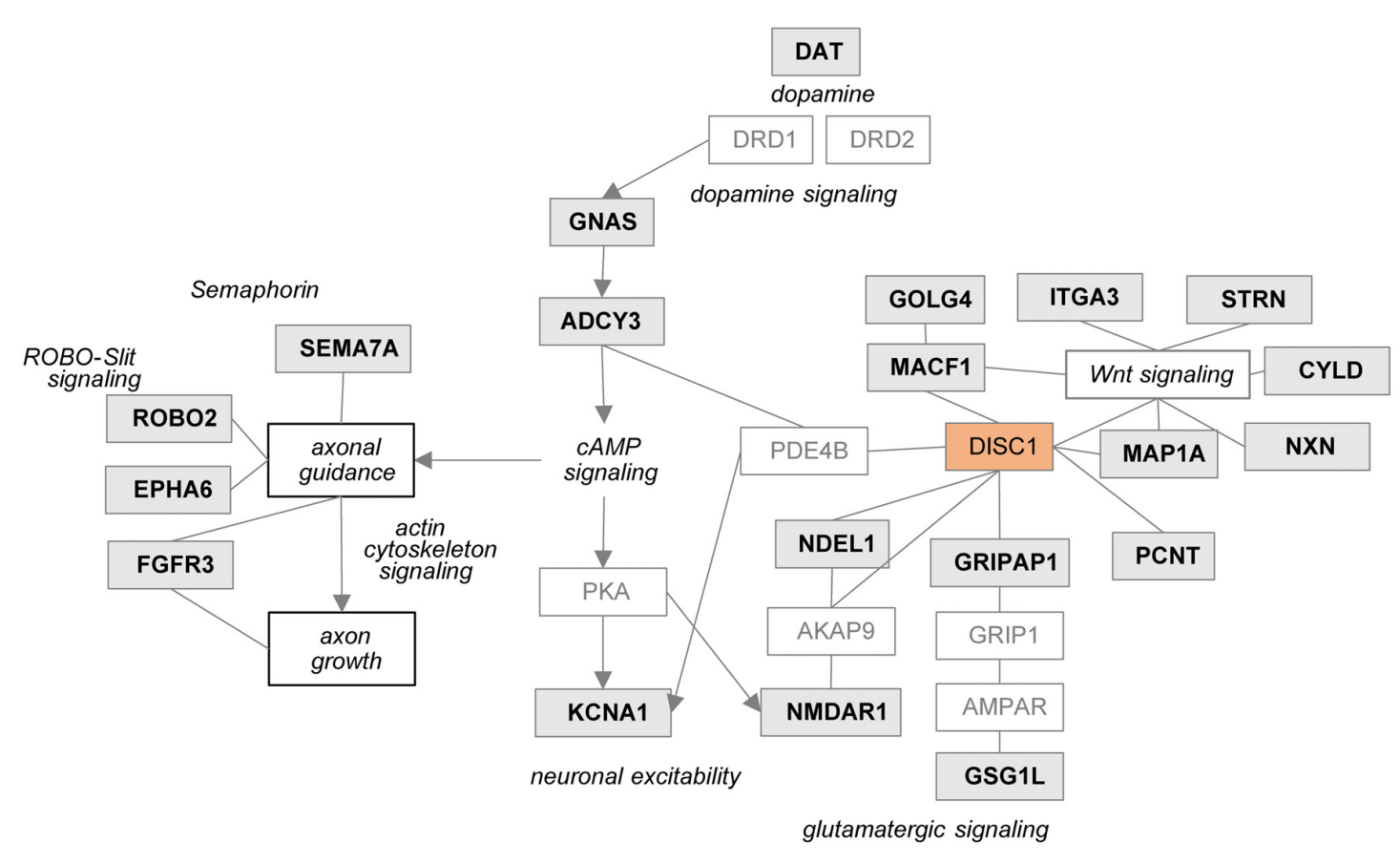

FIGURE 5 | Potential relationship between the DISC1 regulated proteins and synaptic processes. Proteins regulated in tgDISC1 rats from this study are marked in gray boxes.

The protein level changes of this enzyme along with the corresponding G-protein GNAS observed herein supports previous studies proposing dysregulation of cAMP signaling by DISC1 (Millar et al., 2005; Kvajo et al., 2011; Crabtree et al., 2017).

In a mouse Disc1 mutant model, functional reduction of Kv1.1/KCNA1 was proposed to contribute to alterations in neuronal excitability and short-term plasticity. Reduction of this channel was accompanied by reduced phosphodiesterase 4 activity and elevated cAMP levels in the PFC of Disc1 mutant mice (Crabtree et al., 2017). Interestingly, in our DISC1 overexpressing transgenic model, we found an increase of this and several proteins in the voltage-gated potassium channel complex suggesting potential dyregulation of electrophysiological synaptic functions (Supplementary Figure S8).

Current data also revealed that proteins associated with axonal guidance pathways were altered by DISC1 overexpression: the axonal guidance receptors semaphorin 7A (SEMA7A), $\mathrm{EPH}$ receptor A6 (EPHA6), roundabout receptor 2 (ROBO2), fibroblast growth factor receptor 3 (FGFR3) and integrin subunit alpha 3/very late activation protein 3 receptor, alpha-3 subunit (ITGA3) were shown to be modulated by DISC1 (Figure 3B). The leading edge of the axons contains receptors that sense guidance cues and aid in the navigation and migration of axons. The attraction or repulsion of cues promotes or decreases active actin polymerization, resulting in axonal extension or retraction by triggering the actin cytoskeleton signaling and Rho-GTPase pathways, as also proposed in the current pathway enrichment analysis (reviewed in Dent et al., 2011; Spillane and Gallo, 2014; Van Battum et al., 2015). The receptor SEMA7A stimulates axonal growth through integrins and MAPK signaling (Pasterkamp et al., 2003). The roundabout receptor 2 , $\mathrm{ROBO} 2$ is the main receptor from the Slit-Robo pathway, that is involved in axon guidance and which is also associated with DISC1-interacting proteins SRGAP2 and 3 (Camargo et al., 2007). The Ephrin receptor signaling pathway, predicted to be regulated by DISC1, is critical for embryonic development and known as a mediator of axon guidance (Kvajo et al., 2011).

In perspective, alterations of these developmental pathways and processes could explain the subtle neurodevelopmental phenotypes in the tgDISC1, where the substantia nigra (SN) contains fewer dopaminergic neurons (DA), fewer projections into dorsal striatum, and a shift in the parvalbumin-positive interneurons (Hamburg et al., 2016). DA homeostasis deficiency and the proposed disturbed dopaminergic signaling could explain the observed decrease of DA neurons in the SN. The disturbed axonal guidance signaling could lead to the reduction of the projections into the dorsal striatum and the shift of the parvalbuminpositive interneurons. As protein profiles were obtained from adult tgDISC1 rats, it would be interesting to follow up by studying the profiles in the developing brain to reveal the etiopathology effects of DISC1 which exceeds the scope of this study.

Bioinformatics analyses from the current study predicted that the following biological processes were activated by overexpression of DISC1, i.e., regulation of cell quantities, neuronal and axonal extension and long term potentiation 
(Figure 4). These results may be relevant for interpretation of previous as well as for the design of future studies on DISC1.

\section{CONCLUSION}

Our results suggest that overexpression and/or aberrant DISC1 proteostasis can lead to profound changes in protein networks relevant for mental disorders or endophenotypes and may signify a role for the DISC1 protein alone-in the absence of mutations - in behavioral and neural processes and disorders. DISC1 expression levels likely have to be controlled in a narrow expression window in order to execute adaptive behavior. These findings make the DISC1 protein and its posttranslational modifications a molecular convergence point or sensor for environmental interactions such as oxidative stress. The findings also strongly support the earlier literature indicating involvement of the dopaminergic systems, particularly in the dorsal striatum in functional properties of the DISC1 protein.

\section{AUTHOR CONTRIBUTIONS}

FJS, SVT, CK, JPH, MASS and GL conceived and designed the experiments. FJS, A-LW, BF and MK performed experiments.

\section{REFERENCES}

Atkin, T. A., Brandon, N. J., and Kittler, J. T. (2012). Disrupted in Schizophrenia 1 forms pathological aggresomes that disrupt its function in intracellular transport. Hum. Mol. Genet. 21, 2017-2028. doi: 10.1093/hmg/dds018

Bindea, G., Mlecnik, B., Hackl, H., Charoentong, P., Tosolini, M., Kirilovsky, A., et al. (2009). ClueGO: a Cytoscape plug-in to decipher functionally grouped gene ontology and pathway annotation networks. Bioinformatics 25, 1091-1093. doi: 10.1093/bioinformatics/btp101

Boxall, R., Porteous, D. J., and Thomson, P. A. (2011). DISC1 and Huntington's disease-overlapping pathways of vulnerability to neurological disorder? PLoS One 6:e16263. doi: 10.1371/journal.pone.0016263

Bradshaw, N. J., and Porteous, D. J. (2012). DISC1-binding proteins in neural development, signalling and schizophrenia. Neuropharmacology 62, 1230-1241. doi: 10.1016/j.neuropharm.2010.12.027

Brandon, N. J., and Sawa, A. (2011). Linking neurodevelopmental and synaptic theories of mental illness through DISC1. Nat. Rev. Neurosci. 12, 707-722. doi: $10.1038 / \mathrm{nrn} 3120$

Camargo, L. M., Collura, V., Rain, J. C., Mizuguchi, K., Hermjakob, H., Kerrien, S., et al. (2007). Disrupted in Schizophrenia 1 Interactome: evidence for the close connectivity of risk genes and a potential synaptic basis for schizophrenia. Mol. Psychiatry 12, 74-86. doi: 10.1038/sj.mp.4001880

Chubb, J. E., Bradshaw, N. J., Soares, D. C., Porteous, D. J., and Millar, J. K. (2008). The DISC locus in psychiatric illness. Mol. Psychiatry 13, 36-64. doi: 10.1038/sj. mp.4002106

Crabtree, G. W., Sun, Z., Kvajo, M., Broek, J. A., Fenelon, K., McKellar, H., et al. (2017). Alteration of neuronal excitability and short-term synaptic plasticity in the prefrontal cortex of a mouse model of mental illness. J. Neurosci. 37, 4158-4180. doi: 10.1523/JNEUROSCI.4345-15.2017

Dahoun, T., Trossbach, S. V., Brandon, N. J., Korth, C., and Howes, O. D. (2017). The impact of Disrupted-in-Schizophrenia 1 (DISC1) on the dopaminergic system: a systematic review. Transl. Psychiatry 7:e1015. doi: 10.1038/tp. 2016.282

Davis, A. P., Grondin, C. J., Lennon-Hopkins, K., Saraceni-Richards, C., Sciaky, D., King, B. L., et al. (2015). The comparative toxicogenomics database's 10th year anniversary: update 2015. Nucleic Acids Res. 43, D914-D920. doi: 10.1093/nar/gku935
FJS and RS collected data and processed them. FJS, CK, JPH, MASS and GL interpreted the results. FJS, CK, JPH, MASS and GL wrote the article. CK, JPH, MASS and GL revised the intellectual content.

\section{FUNDING}

MASS was supported by a Heisenberg Fellowship SO 1032/5-1, MASS, CK and BF were supported by EU-FP7 MC-ITN IN-SENS \#607616 grant, MASS, CK and A-LW by a Grant of the Medical Faculty of the Heinrich Heine University Düsseldorf (\#9772569).

\section{ACKNOWLEDGMENTS}

We thank Jovana Malikovic, Dr. Judith Wackerlig and Dr. Gabriela Bindea for their excellent technical assistance and experimental advice.

\section{SUPPLEMENTARY MATERIAL}

The Supplementary Material for this article can be found online at: https://www.frontiersin.org/articles/10.3389/fnmol. 2018.00026/full\#supplementary-material

Dent, E. W., Gupton, S. L., and Gertler, F. B. (2011). The growth cone cytoskeleton in axon outgrowth and guidance. Cold Spring Harb. Perspect. Biol. 3:a001800. doi: $10.1101 /$ cshperspect.a001800

Devine, M. J., Norkett, R., and Kittler, J. T. (2016). DISC1 is a coordinator of intracellular trafficking to shape neuronal development and connectivity. J. Physiol. 594, 5459-5469. doi: 10.1113/JP272187

Gilar, M., Olivova, P., Daly, A. E., and Gebler, J. C. (2005). Two-dimensional separation of peptides using RP-RP-HPLC system with different $\mathrm{pH}$ in first and second separation dimensions. J. Sep. Sci. 28, 1694-1703. doi: 10.1002/jssc. 200500116

Guipponi, M., Santoni, F. A., Setola, V., Gehrig, C., Rotharmel, M., Cuenca, M., et al. (2014). Exome sequencing in 53 sporadic cases of schizophrenia identifies 18 putative candidate genes. PLoS One 9:e112745. doi: 10.1371/journal.pone. 0112745

Hahn, C. G., Banerjee, A., Macdonald, M. L., Cho, D. S., Kamins, J., Nie, Z., et al. (2009). The post-synaptic density of human postmortem brain tissues: an experimental study paradigm for neuropsychiatric illnesses. PLoS One 4:e5251. doi: 10.1371/journal.pone.0005251

Hamburg, H., Trossbach, S. V., Bader, V., Chwiesko, C., Kipar, A., Sauvage, M., et al. (2016). Simultaneous effects on parvalbumin-positive interneuron and dopaminergic system development in a transgenic rat model for sporadic schizophrenia. Sci. Rep. 6:34946. doi: 10.1038/srep 34946

Hayashi-Takagi, A., Takaki, M., Graziane, N., Seshadri, S., Murdoch, H., Dunlop, A. J., et al. (2010). Disrupted-in-Schizophrenia 1 (DISC1) regulates spines of the glutamate synapse via Rac1. Nat. Neurosci. 13, 327-332. doi: $10.1038 / \mathrm{nn} .2487$

Hennah, W., Varilo, T., Kestilä, M., Paunio, T., Arajärvi, R., Haukka, J., et al. (2003). Haplotype transmission analysis provides evidence of association for DISC1 to schizophrenia and suggests sex-dependent effects. Hum. Mol. Genet. 12, 3151-3159. doi: 10.1093/hmg/ddg341

Huntley, R. P., Sawford, T., Mutowo-Meullenet, P., Shypitsyna, A., Bonilla, C., Martin, M. J., et al. (2015). The GOA database: gene Ontology annotation updates for 2015. Nucleic Acids Res. 43, D1057-D1063. doi: 10.1093/nar/gku1113

Jaros, J. A., Rahmoune, H., Wesseling, H., Leweke, F. M., Ozcan, S., Guest, P. C., et al. (2015). Effects of olanzapine on serum protein phosphorylation 
patterns in patients with schizophrenia. Proteomics Clin. Appl. 9, 907-916. doi: 10.1002/prca.201400148

Koide, T., Banno, M., Aleksic, B., Yamashita, S., Kikuchi, T., Kohmura, K., et al. (2012). Common variants in MAGI2 gene are associated with increased risk for cognitive impairment in schizophrenic patients. PLoS One 7:e36836. doi: 10.1371/journal.pone.0036836

Kvajo, M., McKellar, H., Drew, L. J., Lepagnol-Bestel, A. M., Xiao, L., Levy, R. J., et al. (2011). Altered axonal targeting and short-term plasticity in the hippocampus of Disc1 mutant mice. Proc. Natl. Acad. Sci. U S A 108, E1349-E1358. doi: 10.1073/pnas.1114113108

Leliveld, S. R., Bader, V., Hendriks, P., Prikulis, I., Sajnani, G., Requena, J. R., et al. (2008). Insolubility of disrupted-in-schizophrenia 1 disrupts oligomer-dependent interactions with nuclear distribution element 1 and is associated with sporadic mental disease. J. Neurosci. 28, 3839-3845. doi: 10.1523/JNEUROSCI.5389-07.2008

Li, J., Yoshikawa, A., Brennan, M. D., Ramsey, T. L., and Meltzer, H. Y. (2017). Genetic predictors of antipsychotic response to lurasidone identified in a genome wide association study and by schizophrenia risk genes. Schizophr. Res. doi: 10.1016/j.schres.2017.04.009 [Epub ahead of print].

Mateos, J. J., Lomeña, F., Parellada, E., Font, M., Fernández, E., Pavia, J., et al. (2006). Striatal dopamine transporter density decrease in first episode schizophrenic patients treated with risperidone. Rev. Esp. Med. Nucl. 25, 159-165. doi: 10.1157/13088411

Millar, J. K., Christie, S., and Porteous, D. J. (2003). Yeast two-hybrid screens implicate DISC1 in brain development and function. Biochem. Biophys. Res. Commun. 311, 1019-1025. doi: 10.1016/j.bbrc.2003.10.101

Millar, J. K., Pickard, B. S., Mackie, S., James, R., Christie, S., Buchanan, S. R., et al. (2005). DISC1 and PDE4B are interacting genetic factors in schizophrenia that regulate cAMP signaling. Science 310, 1187-1191. doi: 10.1126/science.1112915

Millar, J. K., Wilson-Annan, J. C., Anderson, S., Christie, S., Taylor, M. S., Semple, C. A., et al. (2000). Disruption of two novel genes by a translocation co-segregating with schizophrenia. Hum. Mol. Genet. 9, 1415-1423. doi: 10.1093/hmg/9.9.1415

Ottis, P., Bader, V., Trossbach, S. V., Kretzschmar, H., Michel, M., Leliveld, S. R., et al. (2011). Convergence of two independent mental disease genes on the protein level: recruitment of dysbindin to cell- invasive disrupted- inschizophrenia 1 aggresomes. Biol. Psychiatry 70, 604-610. doi: 10.1016/j. biopsych.2011.03.027

Ow, S. Y., Salim, M., Noirel, J., Evans, C., and Wright, P. C. (2011). Minimising iTRAQ ratio compression through understanding LC-MS elution dependence and high- resolution HILIC fractionation. Proteomics 11, 2341-2346. doi: 10.1002/pmic. 201000752

Pascovici, D., Handler, D. C., Wu, J. X., and Haynes, P. A. (2016). Multiple testing corrections in quantitative proteomics: a useful but blunt tool. Proteomics 16, 2448-2453. doi: 10.1002/pmic.201600044

Pasterkamp, R. J., Peschon, J. J., Spriggs, M. K., and Kolodkin, A. L. (2003). Semaphorin 7A promotes axon outgrowth through integrins and MAPKs. Nature 424, 398-405. doi: 10.1038/nature01790

Phillips, G. R., Huang, J. K., Wang, Y., Tanaka, H., Shapiro, L., Zhang, W., et al. (2001). The presynaptic particle web: ultrastructure, composition, dissolution, and reconstitution. Neuron 32, 63-77. doi: 10.1016/S0896-6273(01)00450-0

Ramsey, A. J., Milenkovic, M., Oliveira, A. F., Escobedo-Lozoya, Y., Seshadri, S., Salahpour, A., et al. (2011). Impaired NMDA receptor transmission alters striatal synapses and DISC1 protein in an age-dependent manner. Proc. Natl. Acad. Sci. U S A 108, 5795-5800. doi: 10.1073/pnas. 1012621108

Sachs, N. A., Sawa, A., Holmes, S. E., Ross, C. A., DeLisi, L. E., and Margolis, R. L. (2005). A frameshift mutation in Disrupted in Schizophrenia 1 in an American family with schizophrenia and schizoaffective disorder. Mol. Psychiatry 10, 758-764. doi: 10.1038/sj.mp.4001667

Sialana, F. J., Gulyassy, P., Májek, P., Sjöstedt, E., Kis, V., Müller, A. C., et al. (2016). Mass spectrometric analysis of synaptosomal membrane preparations for the determination of brain receptors, transporters and channels. Proteomics 16, 2911-2920. doi: 10.1002/pmic.201600234

Silberberg, G., Darvasi, A., Pinkas-Kramarski, R., and Navon, R. (2006). The involvement of ErbB4 with schizophrenia: association and expression studies. Am. J. Med. Genet. B Neuropsychiatr. Genet. 141B, 142-148. doi: 10.1002/ajmg. b. 30275
Spillane, M., and Gallo, G. (2014). Involvement of Rho-family GTPases in axon branching. Small GTPases 5:e27974. doi: 10.4161/sgtp.27974

Stojanovic, T., Orlova, M., Sialana, F. J., Höger, H., Stuchlik, S., Milenkovic, I., et al. (2017). Validation of dopamine receptor DRD1 and DRD2 antibodies using receptor deficient mice. Amino Acids 49, 1101-1109. doi: 10.1007/s00726-0172408-3

Teng, S., Thomson, P. A., McCarthy, S., Kramer, M., Muller, S., Lihm, J., et al. (2017). Rare disruptive variants in the DISC1 Interactome and Regulome: association with cognitive ability and schizophrenia. Mol. Psychiatry doi: 10.1038/mp.2017.115 [Epub ahead of print].

Thomson, P. A., Malavasi, E. L., Grünewald, E., Soares, D. C., Borkowska, M., and Millar, J. K. (2013). DISC1 genetics, biology and psychiatric illness. Front. Biol. 8, 1-31. doi: 10.1007/s11515-012-1254-7

Trossbach, S. V., Bader, V., Hecher, L., Pum, M. E., Masoud, S. T., Prikulis, I., et al. (2016). Misassembly of full-length disrupted-in-Schizophrenia 1 protein is linked to altered dopamine homeostasis and behavioral deficits. Mol. Psychiatry 21, 1561-1572. doi: 10.1038/mp.2015.194

Tyanova, S., Temu, T., Sinitcyn, P., Carlson, A., Hein, M. Y., Geiger, T., et al. (2016). The Perseus computational platform for comprehensive analysis of (prote)omics data. Nat. Methods 13, 731-740. doi: 10.1038/nmeth.3901

Van Battum, E. Y., Brignani, S., and Pasterkamp, R. J. (2015). Axon guidance proteins in neurological disorders. Lancet Neurol. 14, 532-546. doi: 10.1016/S1474-4422(14)70257-1

Vizcaíno, J. A., Deutsch, E. W., Wang, R., Csordas, A., Reisinger, F., Ríos, D., et al. (2014). ProteomeXchange provides globally coordinated proteomics data submission and dissemination. Nat. Biotechnol. 32, 223-226. doi: 10.1038/ nbt.2839

Wang, A.-L., Fazari, B., Chao, O. Y., Nikolaus, S., Trossbach, S. V., Korth, C., et al. (2017). Intra-nasal dopamine alleviates cognitive deficits in tgDISC1 rats which overexpress the human DISC1 gene. Neurobiol. Learn. Mem. 146, 12-20. doi: 10.1016/j.nlm.2017.10.015

Welinder, C., and Ekblad, L. (2011). Coomassie staining as loading control in Western blot analysis. J. Proteome Res. 10, 1416-1419. doi: 10.1021/pr1011476

Wisniewski, J. R., Zougman, A., Nagaraj, N., and Mann, M. (2009). Universal sample preparation method for proteome analysis. Nat. Methods 6, 359-362. doi: 10.1038/nmeth.1322

Xu, B., Roos, J. L., Dexheimer, P., Boone, B., Plummer, B., Levy, S., et al. (2011). Exome sequencing supports a de novo mutational paradigm for schizophrenia. Nat. Genet. 43, 864-868. doi: 10.1038/ng.902

Ye, B., Liao, D., Zhang, X., Zhang, P., Dong, H., and Huganir, R. L. (2000). GRASP-1: a neuronal RasGEF associated with the AMPA receptor/GRIP complex. Neuron 26, 603-617. doi: 10.1016/S0896-6273(00)81198-8

Yerabham, A. S. K., Mas, P. J., Decker, C., Soares, D. C., Weiergräber, O. H., Nagel-Steger, L., et al. (2017). A structural organization for the Disrupted in Schizophrenia 1 protein, identified by high-throughput screening, reveals distinctly folded regions, which are bisected by mental illnessrelated mutations. J. Biol. Chem. 292, 6468-6477. doi: 10.1074/jbc.M116. 773903

Yerabham, A. S., Weiergräber, O. H., Bradshaw, N. J., and Korth, C. (2013). Revisiting disrupted-in-schizophrenia 1 as a scaffold protein. Biol. Chem. 394, 1425-1437. doi: 10.1515/hsz-2013-0178

Zhang, N., Zhong, P., Shin, S. M., Metallo, J., Danielson, E., Olsen, C. M., et al. (2015). S-SCAM, a rare copy number variation gene, induces schizophreniarelated endophenotypes in transgenic mouse model. J. Neurosci. 35, 1892-1904. doi: 10.1523/JNEUROSCI.3658-14.2015

Conflict of Interest Statement: The authors declare that the research was conducted in the absence of any commercial or financial relationships that could be construed as a potential conflict of interest.

Copyright (c) 2018 Sialana, Wang, Fazari, Kristofova, Smidak, Trossbach, Korth, Huston, de Souza Silva and Lubec. This is an open-access article distributed under the terms of the Creative Commons Attribution License (CC BY). The use, distribution or reproduction in other forums is permitted, provided the original author(s) and the copyright owner are credited and that the original publication in this journal is cited, in accordance with accepted academic practice. No use, distribution or reproduction is permitted which does not comply with these terms. 